\title{
Enhanced Healing of Rat Calvarial Bone Defects with Hypoxic Conditioned Medium from Mesenchymal Stem Cells through Increased Endogenous Stem Cell Migration via Regulation of ICAM-1 Targeted- microRNA-221
}

\author{
Woochul Chang ${ }^{2,10}$, Ran Kim ${ }^{2,10}$, Sang In Park', Yu Jin Jung ${ }^{3}$, Onju Ham, Jihyun Lee ${ }^{2}$, Ji Hyeong Kim², \\ Sekyung $\mathrm{Oh}^{5}$, Min Young Lee ${ }^{6}$, Jongmin Kim ${ }^{7}$, Moon-Seo Park ${ }^{2}$, Yong-An Chung ${ }^{1}$, Ki-Chul Hwang ${ }^{8,9}$, and \\ Lee-So Maeng ${ }^{1 \text { ** }}$
}

The use of conditioned medium from mesenchymal stem cells may be a feasible approach for regeneration of bone defects through secretion of various components of mesenchymal stem cells such as cytokines, chemokines, and growth factors. Mesenchymal stem cells secrete and accumulate multiple factors in conditioned medium under specific physiological conditions. In this study, we investigated whether the conditioned medium collected under hypoxic condition could effectively influence bone regeneration through enhanced migration and adhesion of endogenous mesenchymal stem cells. Cell migration and adhesion abilities were increased through overexpression of intercellular adhesion molecule-1 in hypoxic conditioned medium treated group. Intercellular adhesion molecule-1 was upregulated by microRNA-221 in mesenchymal

${ }^{1}$ Institute of Catholic Integrative Medicine, Incheon St. Mary's Hospital, The Catholic University of Korea, College of Medicine, Incheon 403-720, Korea, ${ }^{2}$ Department of Biology Education, College of Education, Pusan National University, Busan 609-735, Korea, ${ }^{3}$ EIT/LOFUS Research Center, International St. Mary's Hospital, Catholic Kwandong University, Incheon 404-834, Korea, ${ }^{4}$ Brain Korea 21 PLUS Project for Medical Science, Yonsei University College of Medicine, Seoul 120-752, Korea, ${ }^{5}$ Department of Neurology and Neurological Sciences, Stanford University School of Medicine, Stanford, CA 94305, USA, ${ }^{6}$ Department of Molecular Physiology, College of Pharmacy, Kyungpook National University, Daegu 702-701, Korea, ${ }^{7}$ Department of Life Systems, Sookmyung Women's University, Seoul 140-742, Korea, ${ }^{8}$ Institute for Bio-Medical Convergence, College of Medicine, Catholic Kwandong University, Gangwon 210-701, Korea, ${ }^{9}$ Catholic Kwandong University International, St. Mary's Hospital, Incheon 404-834, Korea, ${ }^{10}$ These authors contributed equally to this work.

*Correspondence: mls@ catholic.ac.kr

Received 23 February, 2015; revised 22 April, 2015; accepted 24 April, 2015; published online 10 June, 2015

Keywords: bone regeneration, calvarial defect model, hypoxic conditioned medium, intercellular adhesion molecule-1, mesenchymal stem cells, microRNA-221 stem cells because microRNAs are key regulators of various biological functions via gene expression. To investigate the effects in vivo, evaluation of bone regeneration by computed tomography and histological assays revealed that osteogenesis was enhanced in the hypoxic conditioned medium group relative to the other groups. These results suggest that behavioral changes of endogenous mesenchymal stem cells through microRNA-221 targetedintercellular adhesion molecule-1 expression under hypoxic conditions may be a potential treatment for patients with bone defects.

\section{INTRODUCTION}

Regeneration of bone defects caused by tumor resection, inflammation, trauma, and congenital cleft is a complex and wellorchestrated physiologic process of bone induction and conduction, involving a number of cell types and intracellular and extracellular molecular signaling pathways and with definable temporal and spatial sequences that optimize skeletal repair and restore skeletal function. Many clinical trials to stimulate or augment bone regeneration have investigated invasive and non-invasive methods using using biomaterials including enamel matrix (Miron et al., 2014) and biosynthetic scaffolds (Chen et al., 2014b), regenerative medicines (Brägger et al., 1992; Villar and Cochran, 2010), or osteo-inductive reagents and growth factors (Liu et al., 2013a; Srouji et al., 2004).

Mesenchymal stem cells (MSCs) are an ideal seed cell source for effective treatment of bone defects (Vertelov et al., 2013). Transplantation of MSCs is accelerated new bone callus in various preclinical animal models, but with poor survival and differentiation rates. Application of conditioned medium (CM) including paracrine factors from MSCs has recently emerged as an alternative approach to MSC-based therapy (Chen et al., 2008; Kinnaird et al., 2004). Previous studies confirmed that $\mathrm{CM}$ has considerable potential for bone reconstruction without stem cells transplantation. Osugi et al. (2012) demonstrated that CM derived from rat MSCs (rMSCs) can enhance the mi- 
gration and adhesion capability of rMSCs in vitro, and bone augmentation in vivo compared to MSC transplantation. Ando et al. (2014) determined that human MSC-CM promotes the recruitment of murine bone marrow stromal cells and endothelial cells with endothelial progenitor cells. Moreover, they found that these cells established a neo-angiogenic network and restored callus formation. MSCs secrete multiple factors that accumulate in $\mathrm{CM}$ under specific physiological conditions. Normoxic CM (NCM) has been reported to provide tissue protection and regenerative functions via secreted factors such as cytokines, chemokines, and growth factors. NCM injection can also induce stem cell migration into injured tissues in vivo. Hypoxic $\mathrm{CM}(\mathrm{HCM})$ is known to include more factors than NCM (Hung et al., 2007). Previous studies have shown that the factors released from MSCs under hypoxic condition could mitigate tendon damage (Huang et al., 2013) and myocardial infarction through prevention of apoptosis and acceleration of functional recovery (Hwang et al., 2012).

Intercellular adhesion molecule-1 (ICAM-1) is an essential molecule for cell migration and adhesion. A number of studies have revealed that some microRNAs (miRs) can regulate expression of ICAM-1 in several cell types. MiRs are small noncoding single strand RNAs known to function as key molecular regulators of gene expression via post-transcriptional regulation of target mRNA or translational inhibition of target proteins (Jin et al., 2012). Liu et al. (2013b) confirmed that miR-296-3p negatively regulated ICAM-1 by directly targeting its $3^{\prime}$-untranslated region in human prostate cancer tissue. Furthermore, they showed that miR-296-3p increases tumor cell resistance to natural killer cells via downregulation of ICAM-1 expression. Gong et al. (2011) demonstrated that downregula- tion of miR-221 is involved in the overexpression of ICAM-1 protein in epithelial cells following Cryptosporidium parvum infection and its subsequent effects on cell adhesion.

In this study, we investigated the effects of MSC-CM generated under hypoxic and normoxic conditions on endogenous stem cell migration, adhesion, expression of ICAM-1, and miR221 expression. Moreover, the relationship between miR-221 and ICAM-1 expression level was investiqated in vitro and estimated on endogenous MSCs and callus formation in vivo. We demonstrated that HCM from MSCs reinforced ICAM-1 and consequently enhanced bone regeneration through the migration and adhesion ability of endogenous MSCs in a rat calvarial bone defect model. Our results suggest that HCM treatment holds potential to replace the methods currently used, including direct engraftment of artificial structures or injection of MSCs for missing or damaged osseous tissue.

\section{MATERIALS AND METHODS}

Isolation and culture of rat mesenchymal stem cells Primary rMSCs were collected as previously described (Chang et al., 2009). All procedures were approved by the Institutional Animal Care and Use Committee of the Incheon Catholic University Medical School. Bone marrow from rat femurs and tibias was flushed with phosphate-buffered saline (PBS; HyClone, USA) containing $2 \%$ fetal bovine serum (FBS; HyClone). Mononuclear cells were isolated by density gradient centrifugation (Ficoll-Paque Plus; GE Healthcare, Sweden) for $30 \mathrm{~min}$ at $1,000 \mathrm{~g}$, then suspended in fresh Dulbecco's Modified Eagle's Media (DMEM; HyClone) containing $20 \%$ FBS. Cells were then plated at a density of $7 \times 10^{5}$ cells/ well and incubated in a humidified atmosphere of $5 \% \mathrm{CO}_{2}$ at $37^{\circ} \mathrm{C}$.
Preparation of normoxic and hypoxic conditioned medium $\mathrm{NCM}$ and $\mathrm{HCM}$ were obtained as previously reported (Hwang et al., 2012). rMSCs $\left(1 \times 10^{6}\right.$ cells $)$ that were $80 \%$ confluent were re-fed with SFM under normoxic and hypoxic conditions for $12 \mathrm{~h}$. Under hypoxic conditions, rMSCs were incubated at $37^{\circ} \mathrm{C}$ in $5 \% \mathrm{CO}_{2}, 5 \% \mathrm{H}_{2}$ and $0.5 \% \mathrm{O}_{2}$ using a chamber with an anaerobic atmosphere system (Technomart, Korea). The CM was collected after $48 \mathrm{~h}$ of incubation and the cell debris was then removed by centrifugation at $1,000 \times g$ and $4^{\circ} \mathrm{C}$ for $30 \mathrm{~min}$.

\section{Cell migration assay}

Transwell plate with $8 \mu \mathrm{m}$ pore filters (Corning, USA) was used to evaluate the migratory ability of rMSCs. rMSCs $\left(3 \times 10^{4}\right.$ cells) were seeded into the upper chamber with a mixture of SFM, NCM and HCM added to the lower chamber. Following incubation for $12 \mathrm{~h}$ and $24 \mathrm{~h}$ at $37^{\circ} \mathrm{C}$ in $5 \% \mathrm{CO}_{2}, \mathrm{rMSCs}$ that had not migrated through the upper side of filters were scraped off with a cotton wool swab. The filters were then stained with Diff-Quik stain kit (Sysmex, Japan), and the cells that had migrated to the lower side were counted using a light microscope (Nikon Co., Japan) at 100× magnification. The migration assay was conducted in triplicate.

\section{Analysis of cell adhesion and spreadability}

Adhesion and spreadability assays were performed as previously described (Song et al., 2013). To determine the adhesion of rMSCs, $2 \times 10^{4}$ cells were added to each well of 6 -well plates (Corning) and incubated for $12 \mathrm{~h}$. The supernatant was removed and the adherent cells were then fixed in $2 \%$ paraformaldehyde. Photographs of a minimum of five fields of view ( $\times$ 10) were taken of each well, after which cells were counted using the Meta-Morph imaging software, version 7.5 (Molecular Devices, USA). For spreadability analysis, rMSCs were incubated for $12 \mathrm{~h}$ in 2-well plates (Corning) under the conditions described above. Cells were then washed with PBS and fixed in $2 \%$ paraformaldehyde, after which they were stained with Coomassie blue (Santa Cruz Biotechnology, Inc., USA). Stained cells were counted using a light microscope at $100 \times$ magnification. Each experiment was repeated three times.

\section{Real-time polymerase chain reaction analysis}

Total RNA was extracted using an RNeasy mini kit (Qiagen, USA). Reverse transcription was performed using an Omniscript RT kit (Qiagen) with total RNA and oligo(dT) primer (Invitrogen, USA). Total synthesized cDNA templates were re-suspended in 20-fold water and used as templates for real-time polymerase chain reaction (PCR), which was conducted using a MyiQ Single-Color RT-PCR Detection System (TaKaRa, Japan). PCR conditions consisted of initial denaturation at $95^{\circ} \mathrm{C}$ for 5 min, followed by 40 cycles of denaturation at $94^{\circ} \mathrm{C}$ for $10 \mathrm{~s}$, annealing at $60^{\circ} \mathrm{C}$ for $20 \mathrm{~s}$, and extension at $72^{\circ} \mathrm{C}$ for $15 \mathrm{~s}$. A standard denaturation curve was then generated by increasing the temperature in $0.5^{\circ} \mathrm{C}$ increments for 70 cycles. The relative expression of each target gene was calculated using the $\Delta \mathrm{Ct}$ method. The threshold cycle $(\Delta \mathrm{Ct})$ of each target gene was normalized to the cycle number of GAPDH. The following primers were used for mRNA detection: CD44: 5'-CAGGCTCCAGTCATAGTACAAC-3' (forward), 5'-CTTCCAGCTCTCCAGGTAATG-3' (reverse); ICAM-1: 5'-GAGAAGTTGGACAGAACCCTG-3' (forward), 5'-GTTACTTGGTCCCCTTCTGAG-3' (reverse); ITGA-1: 5'-CAGGTCGGGATTGTACAGTATG-3' (forward), 5'-CTGTGTCTATTCCAAGGGCTG-3' (reverse); and GAPDH: 5'-CCATCAACGACCCCTTCATT-3' (forward), 5'GACCAGCTTCCCATTCTCAG-3'(reverse). 
microRNA-linked molecular beacon design

We developed a molecular beacon (MB; Bionics Co., Korea) to detect miR expression in single cells. Specifically, a MB for miR-221 that could form a partially double stranded oligonucleotide was designed. The long oligonucleotide contained the miR-221 sequence and was the perfect reverse complimentary sequence of mature miR-221 (5'-AGCUACAUUGUCUGCUGGGUUUC-3').

\section{Luciferase assay}

The predicted gene targets of miR-221 were identified using a public database (Target Scan; www.targetscan.org). The 3'UTR of ICAM-1, which consisted of 934 base pairs and contained a miR-221 binding site, was synthesized, as was a control sequence containing several mutated bases within the miR221 binding site. Next, the corresponding genes were cloned into the pmirGLO vector. rMSCs were plated at $2.5 \times 10^{4}$ cells/well in 24-well plates (Corning). After $48 \mathrm{~h}$, the pmirGLO vector containing the ICAM-1 binding site for miR-221 was co-transfected with miR-221 or the negative control using Lipofectamine 2000 (Invitrogen). Renilla luciferase was used to normalize cell numbers and transfection efficiency. After an additional $48 \mathrm{~h}$, luciferase activity was measured using the Dual Luciferase assay (Promega Co., USA) according to the manufacturer's instructions. Each assay was repeated three times.

\section{Calvarial bone defect model}

All animal experiments were performed in accordance with the guidelines of the Institutional Animal Care and Use Committee of the Incheon Catholic University Medical School. Eight-weekold male Sprague-Dawley rats ( $n=21,290-300 \mathrm{~g})$ were housed in a light and temperature controlled environment and provided with food and water. The rats were initially anesthetized with $5 \%$ isoflurane in $70 \%$ nitrous oxide and $30 \%$ oxygen using an induction chamber, then maintained by a mixture of $2 \%$ isoflurane under temperature controlled conditions (37 \pm $0.1^{\circ} \mathrm{C}$ ) that were maintained using a rectal thermometer and a heating pad (Harvard Apparatus Inc., USA). The rats were subsequently fixed to a stereotaxic apparatus and the median skin over the head was incised. Next, the periosteum was removed and a $5 \mathrm{~mm}$ calvarial bone defect was created using a trephine hand instrument without inflicting other damage. After the operation, skin closure was accomplished using 4-0 silk suture. Experimental materials were then injected into the defects with greenplast (Green Cross, Korea), which consisted of fibrinogen and thrombin. The fibrinogen was dissolved in $1 \mathrm{ml}$ of SFM, NCM, or HCM, after which it was added to thrombin. The mixed materials filled a volume of $100 \mu$ in the defect. The rats were randomly divided into three groups, SFM, NCM, and HCM.

Computerized tomography analysis of new bone formation Surgical sites were monitored by multidetector CT (Siemens, Germany). Before CT scanning, the rats were anesthetized by an intraperitoneal injection with a combination of zolazepam (10 $\mathrm{mg} / \mathrm{kg}$; Zoletil; Virbac AH, Inc., France) and $2 \%$ xylazine hydrochloride (2 mg/kg; Rumpun; Bayer, Germany), while body temperature was kept at $37^{\circ} \mathrm{C}$ using a heating pad on the scanner bed. Repeated CT imaging was performed once every two weeks at $0,14,28,42$ and 56 days after surgery. Animal scanning was obtained at $5 \mathrm{~mm}$ intervals and a thickness of $5 \mathrm{~mm}$. The CT imaging was transmuted into 3D imaging in each case using the 3D imaging reconstruction software RAPIDIA 2.7 (Infinitt Co., Ltd., Korea). The defect size was measured using the Meta-Morph imaging software version 7.5 (Molecular Devices Inc., USA), after which the area of regenerated bone was compared.

\section{Staining}

Surgical sites were evaluated for new bone formation using three different staining methods. Rats $(n=3)$ were labeled by subcutaneous injection with calcein $(10 \mathrm{mg} / \mathrm{kg}$; Sigma-Aldrich Co., USA) once per week, after which they were anesthetized with $15 \%$ urethane and skulls with defects were removed. The skulls were then fixed with $10 \%$ formalin for $24 \mathrm{~h}$ at $4^{\circ} \mathrm{C}$ and bisected. The bisectional tissue was subsequently embedded in methyl-methacrylate resin and paraffin. Next, the blocks were cut into $4 \mu \mathrm{m}$ sections for hematoxylin and eosin (H\&E) and CD44 staining. The sections were then dewaxed in histoclear (Sigma-Aldrich Co.) and rehydrated through graded alcohol series. After retrieval (Abcam Plc., USA), the sections were incubated overnight with primary antibody at $4^{\circ} \mathrm{C}$ for immunehistochemical staining of CD44. The sections were subsequently incubated with Alexa fluor 546-conjugated anti-lgM secondary antibody (Molecular Probe, USA) and then counterstained with 4',6-diamidino-2-phenylindole (DAPI; Sigma-Aldrich Co.). Finally, the fluorescent images were acquired using a Zeiss LSM510 confocal microscope (Carl Zeiss, Germany).

\section{Statistical analysis}

Samples were compared by one-way ANOVA with post hoc analysis, an independent $t$-test, or the Mann-Whitney $U$ test. Data are presented as the mean \pm the standard deviation and probability values $<0.05$ were considered statistically significant.

\section{RESULTS}

Effect of HCM on rat mesenchymal stem cell migration It has been suggested that MSCs exhibit extensive migration in response to CM (Inukai et al., 2013; Osugi et al., 2012). In this study, the effects of NCM and HCM on migration of rMSCs were evaluated using a transwell assay at $12 \mathrm{~h}$ and $24 \mathrm{~h}$. Treated SFM was used as the negative control. The addition of $\mathrm{NCM}$ and HCM significantly increased the migration of rMSCs at $24 \mathrm{~h}$ relative to that of the treated SFM (Fig. 1A). After $12 \mathrm{~h}$, the miaration rate of rMSC-HCM was 13- and 4.3-fold hiaher than that of SFM and NCM, respectively $\left({ }^{*} p<0.05\right.$ vs. SFM and NCM). After $24 \mathrm{~h}$, the migration rate of $\mathrm{rMSC}-\mathrm{HCM}$ was 30 and 4.3-fold hiaher than that of rMSC-SFM and rMSC-NCM. respectively $\left({ }^{*} p<0.05\right.$ vs SFM and NCM) (Fig. 1B). These results suggest that MSC migration increases via paracrine factors in $\mathrm{CM}$, and that the paracrine effect of $\mathrm{CM}$ is stronger under hypoxic conditions than normoxic conditions.

\section{Potential for HCM to influence rMSC adhesion and spreadability}

To investiaate the potential for $\mathrm{HCM}$ to influence the adhesion and spreadability of MSCs in vitro, rMSCs were treated with $\mathrm{HCM}$ and $\mathrm{NCM}$ for $12 \mathrm{~h}$. As shown in Fig. 2A, the number of rMSCs in the HCM group was considerably higher than in the SFM and NCM groups. Moreover, HCM addition led to a $32 \%$ increase in adhesion relative to SFM and a $22 \%$ increase compared to NCM (Fig. 2B). We measured the spreadability of rMSCs because it is involved in cell adhesion capability. To confirm whether treatment with HCM modulates rMSCs spreadability, rMSCs with each $\mathrm{CM}$ were stained with Coomassie blue and then evaluated under light microscopy. The spreadability of 
A

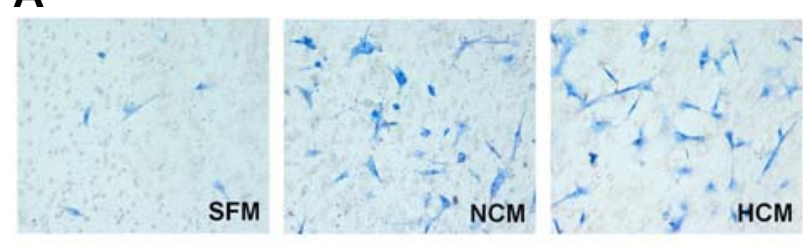

B

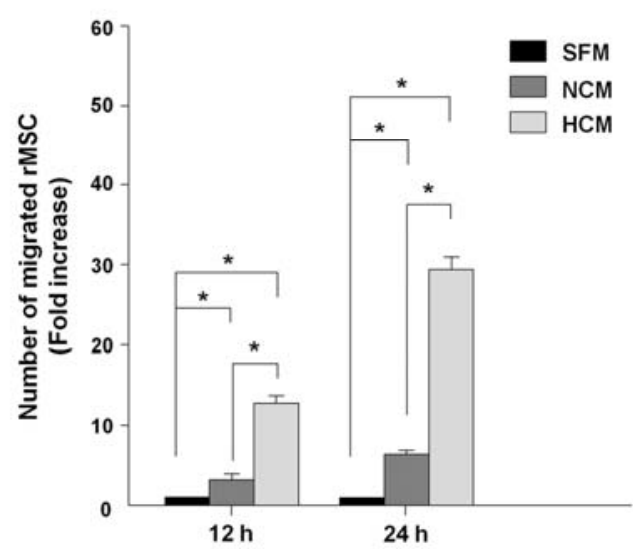

Fig. 1. In vitro migratory ability of CM-treated rMSCs. (A) Migration of rMSCs in response to each medium was measured. Photographs of stained filters show migrated rMSCs at $12 \mathrm{~h}$. (B) Cell migration was compared and evaluated based on microscopic evaluation of five random fields from each stained filter. The migration of rMSCs treated with HCM was significantly enhanced relative to that of rMSCs treated with SFM and NCM. Data are expressed as the mean $\pm \mathrm{SD},{ }^{*} P<0.05$.

rMSCs treated with $\mathrm{HCM}$ was significantly higher than that of the SFM- and NCM-treated rMSCs (Fig. 2C). Specifically, the spreadability of HCM-treated $\mathrm{rMSC}$ s was $75 \%$ greater than that of SFM-treated rMSCs and $25 \%$ greater than that of NCMtreated rMSCs (Fig. 2D). Together, these results suggest that $\mathrm{HCM}$ treatment effectively enhances rMSCs adhesion and spreadability.

HCM enhances ICAM-1 via microRNA-221

We used real-time PCR to measure the mRNA expression of CD44, ICAM-1, and ITGA-1, which play critical roles in the regulation of cell migration and adhesion (Becker et al., 2013; Chen et al., 2015). After $12 \mathrm{~h}$, ICAM-1 expression in the HCMtreated group increased significantly (Fig. 3A). We next evaluated the expression of endogenous miR in rMSCs treated with each CM. miR-221 was selected to target ICAM-1 by Target Scan (http://www.targetscan.org) and downregulated in HCMtreated rMSCs (Figs. 3B-3C). To determine whether miR-221 interacted with the ICAM-1 3'-UTR, a control pmirGLO-ICAM-1 vector was co-transfected with miR-221 or a negative control in rMSCs. In this assay, the expression of ICAM-1 significantly decreased in miR-221-transfected rMSCs (Fig. 3D). In addition, targeting the $3^{\prime}$-UTR of ICAM-1 resulted in decrease of luciferase activity (Fig. 3E). Taken together, these findings indicate that HCM treatment reduces miR-221 expression, consequentially enhances ICAM-1 expression in rMSCs leading to migration and adhesion of rMSC.
A

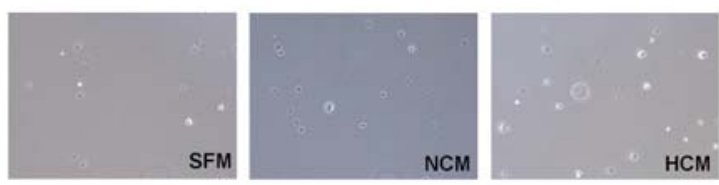

B

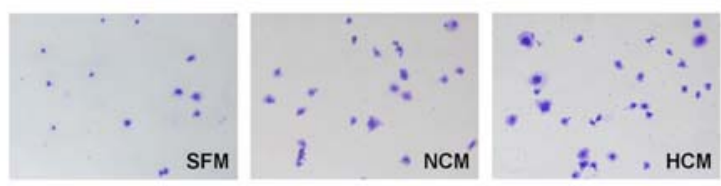

C
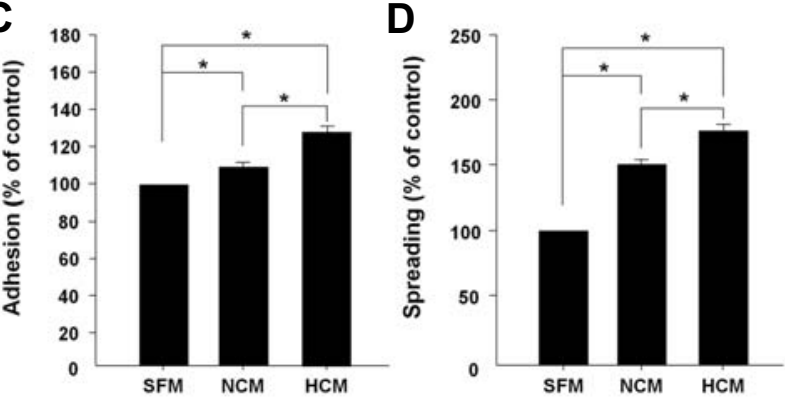

Fig. 2. In vitro adhesion and spreadability of CM-treated rMSCs. (A) Adhesive ability of rMSCs treated with each medium. The media were added when the cells were seeded, and the plates were incubated for $12 \mathrm{~h}$ at $37^{\circ} \mathrm{C}$ under $5 \% \mathrm{CO}_{2}$. After washing, cells were evaluated under a light microscope. (B) Photographs of rMSCs after $12 \mathrm{~h}$. (C, D) Quantitative analysis of adhesive and spreadable cells in each medium. The abilities of rMSCs treated with HCM were significantly increased relative to SFM and NCM. Data are expressed as the mean $\pm \mathrm{SD},{ }^{\star} P<0.05$.

\section{Effect of $\mathrm{HCM}$ on bone regeneration in vivo}

To investigate whether treatment of $\mathrm{HCM}$ improves fractured calvarial bone regeneration, we produced a calvarial bone defect rat model $(n=21)$ to investigate treatment with SFM, NCM, and HCM. CT scanning was performed using a multi-detector CT at $0,14,28,42$, and 56 days after induction of bone defects. During the first 14 days, the CT images showed no changes in defects in any groups; however, considerable bone regeneration was observed after 28 days in the $\mathrm{HCM}$ group relative to the SFM and NCM group (Fig. 4A). As shown in Fig. 4B, the percentage of new bone formation in the NCM and HCM groups was significantly higher than that in the SFM group from 28 to 56 days after $\mathrm{CM}$ treatment. Furthermore, the $\mathrm{HCM}$ treated group showed remarkably higher re-ossification than the NCM-treated group in all CT images. Based on these results, HCM seems to enhance new bone tissue formation in areas with defects.

\section{Histological analysis}

We next evaluated the regenerated bone tissue with immunestaining for H\&E, CD44, and calcein fluorochrome at 56 days after treatment with SFM, NCM, or HCM. The defect sites were stained with $\mathrm{H} \& \mathrm{E}$ for histological examination and quantification of bone regeneration (Sackstein et al., 2008). The arrows in Fig. $5 \mathrm{~A}$ indicate the edges of the host bone, while the dotted lines indicate the area of reconstructed bone tissue. Larger amounts of new bone and connective tissue were observed in 
A

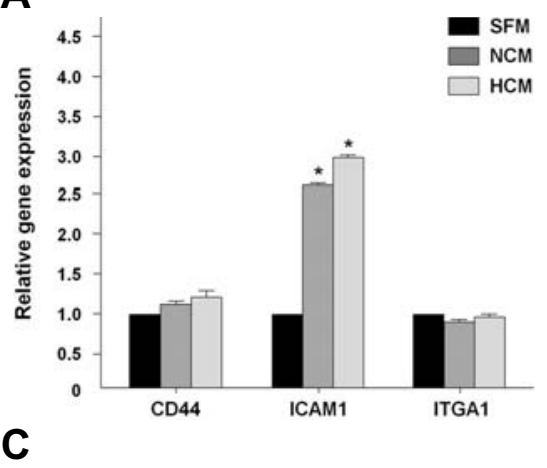

B

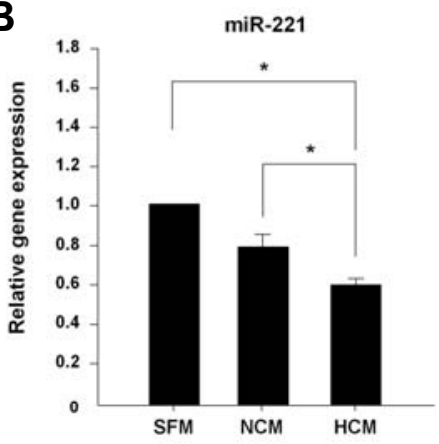

ICAM-1 mRNA

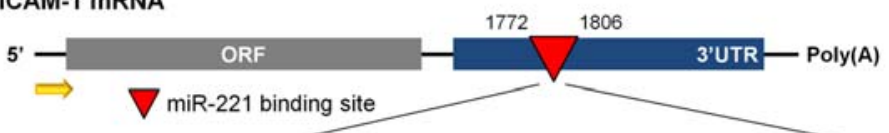

Rat ICAM-1 5' ... GUGGCCUGGGGAUGCAUACUUGUAGCCAGGCCT... 3'

Rno-miR-221

3'...CUUUGGGUCGUCUGUUACAUCGA... 5'

D

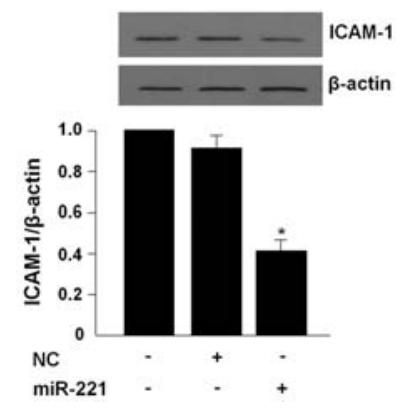

A

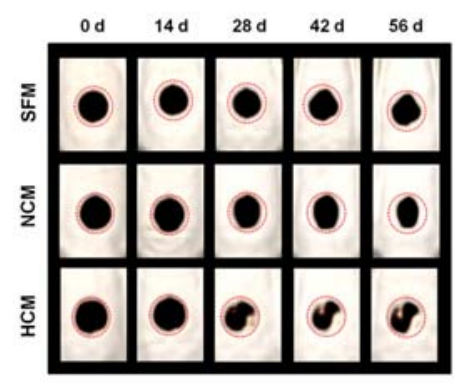

E

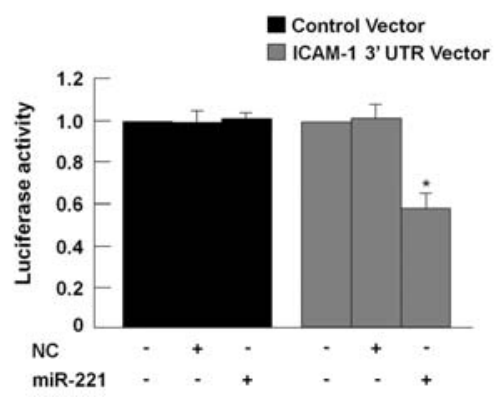

B

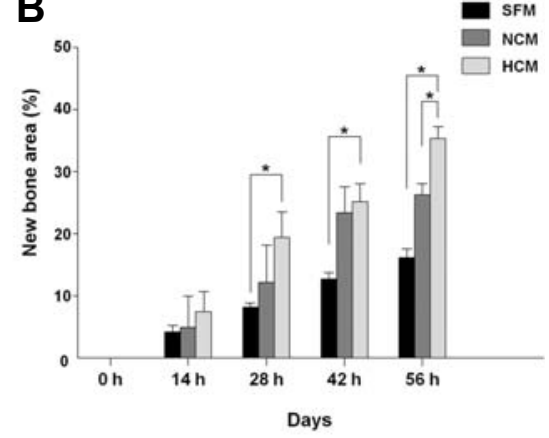

Fig. 3. MiR-221-mediated expression of ICAM1 on rMSCs-treated with CM. (A) Expression of secreted factors of rMSCs in response to $\mathrm{CM}$ for $12 \mathrm{~h}$. The expression of ICAM-1, ITGA-1, and PDGF of rMSCs treated with HCM was significantly higher than that of rMSCs in the other treatment groups. (B) Expression of miR221 was downregulated in rMSCs by $\mathrm{HCM}$ treatment. (C) The sequence of miR-221 showed that it can bind to regulate ICAM-1 expression. (C) Transfection of miR-221 led to decreased ICAM-1 expression. (D) Luciferase assay revealed that ICAM-1 was regulated by miR-221. Data are expressed as the mean \pm SD, ${ }^{\star} P<0.05$.
Fig. 4. CT scanning of bone remodeling after injection of $\mathrm{CM}$ into a calvarial bone defect site in vivo. (A) CT images were taken at $0,14,28$, 42 , and 56 days. (B) The area of regenerated bone in the HCM treatment group was significantly greater than that of the SFM and NCM treatment groups. Data are expressed as the mean $\pm \mathrm{SD},{ }^{*} P<0.05$. the NCM- and HCM-treated groups than in the SFM-treated group. Moreover, the areas of newly reconstructed bone and connective tissue in the HCM group were larger and thicker than in the NCM group (Fig. 5A). Defected region were immunostained for CD44, a specific marker of MSCs, to detect endogenous MSCs in newly mineralized bone tissue (Maurel et al., 2014; Osugi et al., 2012). The arrows in the figure indicate CD44 expression, which was identified by red staining. Cell nuclei were labeled with DAPI (blue). The HCM group showed a greater concentration of CD44-displayed MSCs in all areas of the fracture compared to the other groups (Fig. 5B). Specifically, there were $25 \%$ and $12 \%$ more CD44-labeled cells in the HCM treatment group than in the SFM and NCM groups, respectively $\left({ }^{*} p<0.05\right.$ vs. SFM and NCM) (Fig. 5D). Fluorochrome-based analysis following calcein staining was used to visualize calcified bone structures and differentiate new bone tissue formation. Specifically, each group was intravenously injected with the fluorochrome calcein twice during the two weeks before sacrifice, after which the fluorescence was evaluated. The newly mineralized area is marked with arrows in the figure, which was 
A

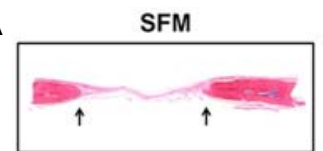

B

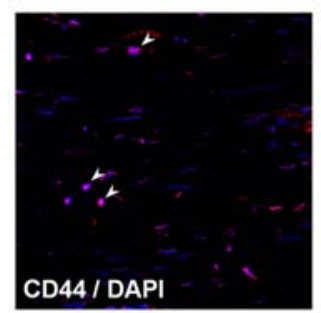

C

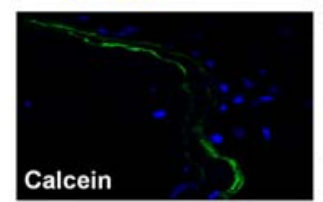

D

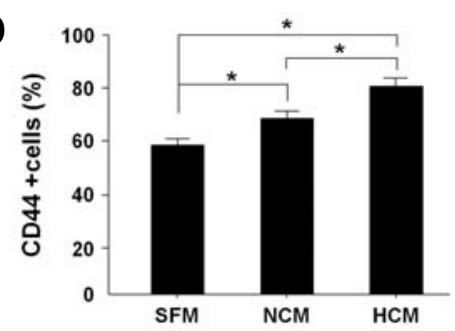

NCM
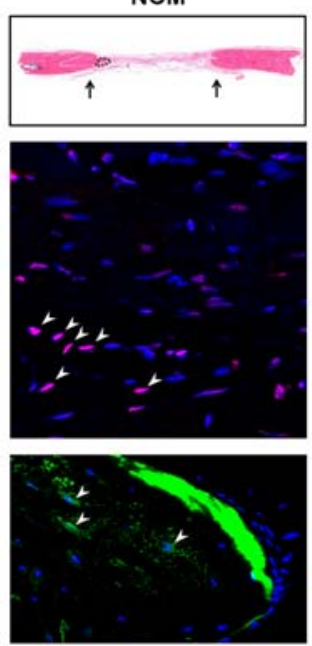
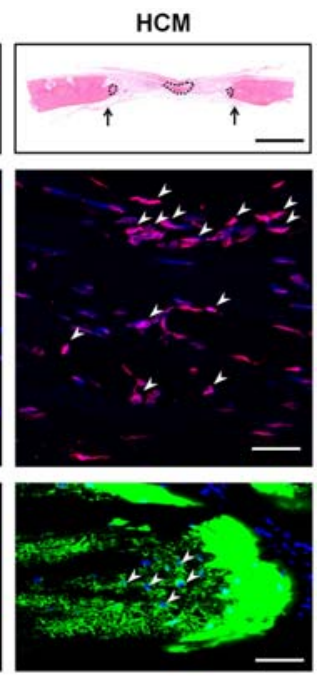

E

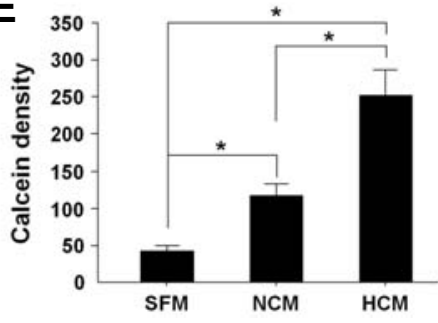

Fig. 5. Histological analysis of regenerated bone tissue after treatment of $\mathrm{CM}$ into calvarial bone defect model in vivo. (A) H\&E staining: arrows indicate the edges of the host bone, and dotted lines indicate reconstructed bone tissue. (B) Immunostaining of CD44: endogenous MSCs were visualized by antiCD44 antibody labeling in the mineralized bone. (C, D) Calcein fluorochrome-based staining: the newly formed cells in the calvarial defect area are presented. Scale bar, $50 \mu \mathrm{m}$. Data are expressed as the mean $\pm \mathrm{SD},{ }^{\star} P<0.05$. more extensive in HCM-rMSCs than in the other groups. Moreover, the cells labeled with calcein were observed in the edges of the host bone (Fig. 5C). The calcein density in the bone defect in the HCM treatment aroup was 5.6- and 2.3-fold hiqher than in the SFM and NCM groups $\left({ }^{*} p<0.05\right.$ vs. SFM and $\mathrm{NCM}$ ) (Fig. 5E). Together, these findings suggest that HCM improves bone regeneration through stimulation of endogenous MSCs via a paracrine interaction in defect site.

\section{DISCUSSION}

When the normal process of bone reconstruction is impairable or insufficient, supportive therapeutic strategies can be used to stimulate and augment bone regeneration (Walsh et al., 2008). MSCs are attractive candidates for cell therapy in impaired bone healing due to their potential for spontaneous proliferation and multi-lineage differentiation (Ham et al., 2014; Sun et al., 2012). MSCs are usually combined with biosynthetic scaffolds to treat bone fractures (Zhou et al., 2011). They have recently garnered considerable attention with their ability to secrete various cytokines, chemokines, and growth factors, thereby acting as trophic mediators that regulate neighboring cells. Some studies have reported that these factors can influence proliferation and differentiation of osteoblasts by forming a sensitive microenvironment (Sun et al., 2012). There is also abundant evidence that a mesenchyme-derived microenvironment can affect the differentiation, proliferation, and function of many other cells through different signaling pathways. For example, Sassoli et al. (2014) investigated the paracrine signaling effects of MSCs on skeletal myoblasts. They confirmed that sphingosine 1phosphate (S1P), secreted by MSCs, stimulated myoblasts and satellite cell proliferation to promote skeletal muscle regeneration. Park et al. (2015) suggested that tonsil-derived MSCs express and secrete Cyclin 1 (CCN1) in response to endodermal differentiation cues. Then CCN1 stimulates the migration and tube formation of human umbilical vein endothelial cells (HUVEC) mediated by integrin $\alpha(v) \beta(3)$ and AMPK. Based on the paracrine effect of MSCs, several studies have investigated the use of $\mathrm{CM}$ as an alternative therapeutic approach to previous methods using MSCs. Growth factors such as transforming growth factor-beta (TGF- $\beta$ ), bone morphogenic protein (BMP), fibroblast growth factor (FGF), platelet-derived growth factor (PDGF), and insulin-like growth factor (IGF) play an important role in bone and cartilage formation, fracture healing, and repair of other musculoskeletal tissues (Lieberman et al., 2002).

This present study demonstrated the links between target gene regulation with $\mathrm{miR}, \mathrm{HCM}$-induced MSCs, and bone regeneration by confirming the response of rMSCs treated with SFM, NCM, or HCM based on cell migration, adhesion, and spreadability. MSC-CM-mediated paracrine factors enhance wound healing through some predominant pathways, as evidenced by the demonstrated action of MSC-CM and secreted molecules (Chen et al., 2014a). One of the mechanisms is MSC-CM may regulate cell migration in response to injury (Smith et al., 2010). Previous studies have demonstrated that MSC-CM treatment enhanced the migration rate of MSCs (Osugi et al., 2012). Our data are consistent with previous reports demonstrating the increased migration with MSC-CM. Meanwhile the present study modified the conditions to obtain rMSC-CM in hypoxia as well as normoxia, and obtained the results that $\mathrm{HCM}$ significantly enhanced the migration of rMSCs, the effects of which increasing in a time-dependent fashion (Fig. 
1). Cell adhesion and spreadability showed a similar tendency (Fig. 2). Both datasets indicate that CM generated under hypoxia increases migration, as well as the adhesion and spreading rates of rMSCs. These results suggest that HCM considerably affects bone regeneration through behavioral changes in rMSCs.

There are some molecules that regulate cell mobilization. The cell adhesion molecule CD44 regulates diverse cellular functions, including cell-cell and cell-matrix interaction, cell motility, migration, differentiation, and growth (Chen et al., 2015). For cellular motility, integrins play roles which are an essential process for embryonic development and tissue morphogenesis, and also for pathological processes such as tumor cell invasion and metastasis (Smerling et al., 2007). ICAM-1 is also associated with the migration and adhesion of various cell types (Bharadwaj et al., 2013; Dong et al., 2014) and regulated by some miRs which are modulators of gene expression at the level of transcription and translation (Gong et al., 2011; Tabet et al., 2014; Ueda et al., 2009; Zuo et al., 2013). Our data showed that the expression of ICAM-1 increased significantly in response to $\mathrm{HCM}$ treatment; therefore, it was selected as a target gene (Fig. 3A). Using Target Scan, miR-221 was identified as a candidate of the target gene regulator that was downregulated by HCM treatment (Fig. 3B). Targeting the $3^{\prime}$-UTR of ICAM-1 resulted in decreased expression of ICAM-1 and luciferase activity in miR-221-transfected cells (Figs. 3C-3E). These results indicate that HCM reduces miR-221 expression and increase ICAM-1 expression, which enhance the migration and adhesion of endogenous rMSCs in wounded sites.

Some studies demonstrated that $\mathrm{CM}$ accelerated wound healing and osteogenesis through multiple reqenerative mechanisms in vivo (Ando et al., 2014; Chen et al., 2008; Osugi et al., 2012; Wang et al., 2012). Graft of MSC-CM showed that the newly regenerated bone bridge almost covered the defected. Interestingly, the effects of MSC-CM were stronger than those of the MSC treatment (Osugi et al., 2012). The secreted factor contained in MSC-CM that recruit BM-MSCs and endothelial cells/endothelial progenitor cells, inhibit inflammation and apoptosis, and promote osteoblast differentiation, anqiogenesis, and cell proliferation in vivo (Ando et al., 2014). The present study modified to take up the previous studies, rats with calvarial fractures were treated with CM and then subjected to CT scanning and histological analysis. The CT scan and staining data revealed more effective osteogenesis whereby the fractured area was filled with more new bone and thicker connective tissue in response to HCM (Figs. 4 and 5A). Immunostaining of defected site with anti-CD44 antibody increased in the HCM group, and the results indicated that $\mathrm{HCM}$ has potential to mobilize endogenous MSCs. (Figs. 5B and 5D). Calcein $\left(\mathrm{C}_{30} \mathrm{H}_{26} \mathrm{~N}_{2} \mathrm{O}_{13}\right)$ has been used to visualize calcified bone structure and investigate bone remodeling or regeneration (Du et al., 2001). In this study, calcein fluorescence was brighter across the defect area in the HCM-treated group than in the other groups, further demonstrating that HCM treatment increases bone regeneration (Figs. $5 \mathrm{C}$ and $5 \mathrm{E})$. These results together indicate that HCM treatment enhances bone regeneration in calvarial bone defects by inducing migration of endogenous MSCs.

In conclusion, the present study demonstrated that reconstruction of bone defects is significantly improved with MSC-CMs generated under hypoxia through enhancement of endogenous MSC migration and adhesion, as well as target gene regulation by $\mathrm{miR}$. CM treatment has proved to have several advantages including minimal invasiveness, efficiency, and cost effectiveness, thereby the potential for further clinical applications for bone defects.

\section{ACKNOWLEDGMENTS}

This research was supported by the Basic Science Research Program through the National Research Foundation (NRF) of Korea funded by the Ministry of Education, Science and Technology (2013R1A1A1008066), the Republic of Korea.

\section{REFERENCES}

Ando, Y., Matsubara, K., Ishikawa, J., Fujio, M., Shohara, R., Hibi, H., Ueda, M., and Yamamoto, A. (2014). Stem cell-conditioned medium accelerates distraction osteogenesis through multiple regenerative mechanisms. Bone 61, 82-90.

Becker, H.M., Rullo, J., Chen, M., Ghazarian, M., Bak, S., Xiao, H., Hay, J.B, and Cybulsky, M.I. (2013). $\alpha 1 \beta 1$ integrin-mediated adhesion inhibits macrophage exit from a peripheral inflammatory lesion. J. Immunol. 190, 4305-4314.

Bharadwaj, A.S., Schewitz-Bowers, L.P., Wei, L., Lee, R.W., and Smith, J.R. (2013). Intercellular adhesion molecule 1 mediates migration of Th1 and Th17 cells across human retinal vascular endothelium. Invest. Ophthalmol. Vis. Sci. 54, 6917-6925.

Brägger, U., Hämmerle, C.H., Mombelli, A., Bürgin, W., and Lang, N.P. (1992). Remodelling of periodontal tissues adjacent to sites treated according to the principles of guided tissue regeneration (GTR). J. Clin. Periodontol. 19, 615-624.

Chang, W., Song, B.W., Lim, S., Song, H., Shim, C.Y., Cha, M.J., Ahn, D.H., Jung, Y.G., Lee, D.H., Chung, J.H., et al. (2009) Mesenchymal stem cells pretreated with delivered Hph-1-Hsp70 protein are protected from hypoxia-mediated cell death and rescue heart functions from myocardial injury. Stem Cells 27, 22832292.

Chen, L., Tredget, E.E., Wu, P.Y., and Wu, Y. (2008). Paracrine factors of mesenchymal stem cells recruit macrophages and endothelial lineage cells and enhance wound healing. PLoS One 3, e1886.

Chen, L., Xu, Y., Zhao, J., Zhang, Z., Yang, R., Xie, J., Liu, X., and Qi, S. (2014a). Conditioned medium from hypoxic bone marrowderived mesenchymal stem cells enhances wound healing in mice. PLoS One 9, e96161.

Chen, Y., Bai, B., Zhang, S., Ye, J., Zhai, H., Chen, Y., Zhang, L., and Zeng, Y. (2014b). Study of a novel three-dimensional scaffold to repair bone defect in rabbit. J. Biomed. Mater. Res. A. 102, 1294-1304.

Chen, X., Ali Khajeh, J., Ju, J.H., Gupta, Y.K., Stanley, C.B., Do, C., Heller, W.T., Aggarwal, A.K., Callaway, D.J., and Bu, Z. (2015). Phosphatidylinositol 4,5-bisphosphate clusters the cell adhesion molecule CD44 and assembles a specific CD44-Ezrin hetero complex, as revealed by small angle neutron scattering. J. Biol. Chem. 290, 6639-6652.

Dong, Z., Fu, S., Xu, X., Yang, Y., Du, L., Li, W., Kan, S., Li, Z., Zhang, X., Wang, L., et al. (2014). Leptin-mediated regulation of ICAM-1 is Rho/ROCK dependent and enhances gastric cancer cell migration. Br. J. Cancer 110, 1801-1810.

Du, SJ., Frenkel, V., Kindschi, G., and Zohar, Y. (2001). Visualizing normal and defective bone development in zebrafish embryos using the fluorescent chromophore calcein. Dev. Biol. 238, 239246.

Gong, A.Y., Hu, G., Zhou, R., Liu, J., Feng, Y., Soukup, G.A., and Chen, X.M. (2011). MicroRNA-221 controls expression of intercellular adhesion molecule-1 in epithelial cells in response to Cryptosporidium parvum infection. Int. J. Parasitol. 41, 397-403.

Ham, O., Lee, C., Song, B.W., Lee, S.Y., Kim, R., Park, J.H., Lee, J., Seo, H., Lee, C.Y., Chung, Y.A., et al. (2014). Upregulation of miR-23b enhances the autologous therapeutic potential for degenerative arthritis by targeting PRKACB in synovial fluidderived mesenchymal stem cells from patients. Mol. Cells 37, 449-456.

Hung, S.C., Pochampally, R.R., Chen, S.C., Hsu, S.C., and Prockop, D.J. (2007). Angiogenic effects of human multipotent stromal cell conditioned medium activate the PI3K-Akt pathway in hypoxic endothelial cells to inhibit apoptosis, increase survival, and stimulate angiogenesis. Stem Cells 25, 2363-2370.

Huang, T.F., Yew, T.L., Chiang, E.R., Ma, H.L., Hsu, C.Y., Hsu, S.H., Hsu, Y.T., and Hung, S.C. (2013). Mesenchymal stem cells from a hypoxic culture improve and engraft Achilles tendon 
repair. Am. J. Sports Med. 41, 1117-1125.

Hwang, H.J., Chang, W., Song, B.W., Song, H., Cha, M.J., Kim, I.K., Lim, S., Choi, E.J., Ham, O., Lee, S.Y., et al. (2012). Antiarrhythmic potential of mesenchymal stem cell is modulated by hypoxic environment. J. Am. Coll. Cardiol. 60, 1698-1706.

Inukai, T., Katagiri, W., Yoshimi, R., Osugi, M., Kawai, T., Hibi, H., and Ueda, M. (2013). Novel application of stem cell-derived factors for periodontal regeneration. Biochem. Biophys. Res. Commun. 430, 763-768.

Jin, H.L., Kim, J.S., Kim, Y.J., Kim, S.J., Broxmeyer, H.E., and Kim, K.S. (2012). Dynamic expression of specific miRNAs during erythroid differentiation of human embryonic stem cells. Mol. Cells 34, 177-183.

Kinnaird, T., Stabile, E., Burnett, M.S., Shou, M., Lee, C.W., Barr, S., Fuchs, S., and Epstein, S.E. (2004). Local delivery of marrowderived stromal cells augments collateral perfusion through paracrine mechanisms. Circulation 109, 1543-1549.

Lieberman, J.R., Daluiski, A., and Einhorn, T.A. (2002). The role of growth factors in the repair of bone. Biology and clinical applications. J. Bone Joint Surg. Am. 84-A, 1032-1044.

Liu, T., Wu, G., Wismeijer, D., Gu, Z, and Liu, Y. (2013a). Deproteinized bovine bone functionalized with the slow delivery of BMP-2 for the repair of critical-sized bone defects in sheep. Bone 56, 110-118.

Liu, X., Chen, Q., Yan, J., Wang, Y., Zhu, C., Chen, C., Zhao, X., Xu, M., Sun, Q., Deng, R., et al. (2013b). MiRNA-296-3p-ICAM1 axis promotes metastasis of prostate cancer by possible enhancing survival of natural killer cell-resistant circulating tumour cells. Cell Death Dis. 4, e928.

Maurel, D.B., Benaitreau, D., Jaffré, C., Toumi, H., Portier, H., Uzbekov, R., Pichon, C., Benhamou, C.L., Lespessailles, E., and Pallu, S. (2014). Effect of the alcohol consumption on osteocyte cell processes: a molecular imaging study. J. Cell Mol. Med. 18, 1680-1693.

Miron, R.J., Wei, L., Bosshardt, D.D., Buser, D., Sculean, A., and Zhang, Y. (2014). Effects of enamel matrix proteins in combination with a bovine-derived natural bone mineral for the repair of bone defects. Clin. Oral Investig. 18, 471-478.

Osugi, M., Katagiri, W., Yoshimi, R., Inukai, T., Hibi, H., and Ueda, M. (2012). Conditioned media from mesenchymal stem cells enhanced bone regeneration in rat calvarial bone defects. Tissue Eng. Part A 18, 1479-1489.

Park, Y.S., Hwang, S., Jin, Y.M., Yu, Y., Jung, S.A., Jung, S.C. Ryu, K.H., Kim, H.S., and Jo, I. (2015). CCN1 secreted by tonsilderived mesenchymal stem cells promotes endothelial cell angiogenesis via integrin $\alpha$ v $\beta 3$ and AMPK. J. Cell Physiol. 230, 140149.

Sackstein, R., Merzaban, J.S., Cain, D.W., Dagia, N.M., Spencer, J.A., Lin, C.P., and Wohlgemuth, R. (2008). Ex vivo glycan engineering of CD44 programs human multipotent mesenchyma stromal cell trafficking to bone. Nat. Med. 14, 181-187.

Sassoli, C., Frati, A., Tani, A., Anderloni, G., Pierucci, F., Matteini, F., Chellini, F., Zecchi Orlandini, S., Formigli, L., and Meacci, E. (2014). Mesenchymal stromal cell secreted sphingosine 1phosphate (S1P) exerts a stimulatory effect on skeletal myoblast proliferation. PLoS One 9, e108662.

Smerling, C., Tang, K., Hofmann, W., and Danker, K. (2007). Role of the alpha(1) integrin cytoplasmic tail in the formation of focal complexes, actin organization, and in the control of cell migration. Exp. Cell Res. 313, 3153-3165.

Smith, A.N., Willis, E., Chan, V.T., Muffley, L.A., Isik, F.F., Gibran, N.S., and Hocking, A.M. (2010). Mesenchymal stem cells induce dermal fibroblast responses to injury. Exp. Cell Res. 316, 48-54.

Song, B.W., Chang, W., Hong, B.K., Kim, I.K., Cha, M.J., Lim, S. Choi, E.J., Ham, O., Lee, S.Y., Lee, C.Y., et al. (2013). Protein kinase $C$ activation stimulates mesenchymal stem cell adhesion through activation of focal adhesion kinase. Cell Transplant. 22, 797-809.

Srouji, S., Blumenfeld, I., Rachmiel, A., and Livne, E. (2004). Bone defect repair in rat tibia by TGF-beta1 and IGF-1 released from hydrogel scaffold. Cell Tissue Bank 5, 223-230.

Sun, J., Zhou, H., Deng, Y., Zhang, Y., Gu, P., Ge, S., and Fan, X (2012). Conditioned medium from bone marrow mesenchymal stem cells transiently retards osteoblast differentiation by downregulating runx2. Cells Tissues Organs 196, 510-522.

Tabet, F., Vickers, K.C., Cuesta Torres, L.F., Wiese, C.B., Shoucri, B.M., Lambert, G., Catherinet, C., Prado-Lourenco, L., Levin, M.G., Thacker, S., et al. (2014). HDL-transferred microRNA-223 regulates ICAM-1 expression in endothelial cells. Nat. Commun. 5, 3292.

Ueda, R., Kohanbash, G., Sasaki, K., Fujita, M., Zhu, X., Kastenhuber, E.R., McDonald, H.A., Potter, D.M., Hamilton, R.L., Lotze, M.T., et al. (2009). Dicer-regulated microRNAs 222 and 339 promote resistance of cancer cells to cytotoxic Tlymphocytes by down-regulation of ICAM-1. Proc. Natl. Acad. Sci. USA 106, 10746-10751.

Vertelov, G., Kharazi, L., Muralidhar, M.G., Sanati, G., Tankovich, T., and Kharazi, A. (2013). High targeted migration of human mesenchymal stem cells grown in hypoxia is associated with enhanced activation of RhoA. Stem Cell Res. Ther. 4, 5 .

Villar, C.C., and Cochran, D.L. (2010). Regeneration of periodontal tissues: guided tissue regeneration. Dent. Clin. North Am. 54, 73-92.

Walsh, W.R., Langdown, A.J., Auld, J.W., Stephens, P., Yu, Y., Vizesi, F., Bruce, W.J., and Pounder, N. (2008). Effect of low intensity pulsed ultrasound on healing of an ulna defect filled with a bone graft substitute. J. Biomed. Mater Res. B Appl. Biomater. 86, 74-81.

Wang, C.Y., Yang, H.B., Hsu, H.S., Chen, L.L., Tsai, C.C., Tsai, K.S., Yew, T.L., Kao, Y.H., and Hung, S.C. (2012). Mesenchymal stem cell-conditioned medium facilitates angiogenesis and fracture healing in diabetic rats. J. Tissue Eng. Regen. Med. 6, 559-569.

Zhou, H., Xiao, C., Wang, Y., Bi, X., Ge, S., and Fan X. (2011). In vivo efficacy of bone marrow stromal cells coated with betatricalcium phosphate for the reconstruction of orbital defects in canines. Invest. Ophthalmol. Vis. Sci. 52, 1735-1741.

Zuo, J., Xia, J., Ju, F., Yan, J., Zhu, A., Jin, S., Shan, T., and Zhou, H. (2013). MicroRNA-148a can regulate run-related transcription factor 3 gene expression via modulation of DNA methyltranferase 1 in gastric cancer. Mol. Cells 35, 313-319. 